\title{
Euglycemic Diabetic Ketoacidosis, diagnostic challenge in resource limited countries: Case series
}

Ameyaw $\mathrm{E}^{1}$

${ }^{1}$ Department of Child Health, School of Medicine and Dentistry, Kwame Nkrumah University of Science and Technology, Kumasi

\begin{abstract}
Euglycemic diabetic ketoacidosis (Euglycemic DKA) can present with diagnostic difficulty in resource limited countries because of absence of hyperglycemia which is a key feature in diagnosis of DKA. Diagnosis can be delayed or even missed entirely with detrimental consequences in resource limited countries because of poor health infrastructure including lack of laboratory support. We describe two children with type 1 diabetes mellitus (T1DM) who presented with infections and euglycemic DKA. One presented with severe malaria and the other with cerebral malaria and urinary tract infection. Both patients were found to have normoglycemia and ketonuria with clinical signs of metabolic acidosis which eventually led to diagnosis of euglycemic DKA for which they were successfully managed. Our account emphasized the need for testing blood glucose as well as urine ketones in critically ill children and adolescents with infections especially if they have been diagnosed with diabetes mellitus. Because the clinical features of euglycemic DKA are similar to those of infections which are rather more common in Ghana and Africa.
\end{abstract}

Key Words: Children, Euglycemic diabetic ketoacidosis, Infections, Resource limited countries

Correspondence email: ekameyaw@yahoo.com

https://orcid.org/0000-0001-5548-9905

This is an open-access article distributed under the terms of the Creative Commons Attribution License, which permits unrestricted use, distribution, and reproduction in any medium, provided the original author and source are credited (CC BY 4.0)

\section{Introduction}

Diabetic ketoacidosis (DKA) is a metabolic emergency in which hyperglycemia is associated with a metabolic acidosis due to increased levels of ketone bodies in the blood. It is characterized by a serum glucose level greater than $250 \mathrm{mg}$ per $\mathrm{dl}$, a blood $\mathrm{pH}$ less than 7.3 , a serum bicarbonate level less than $18 \mathrm{mmol} / \mathrm{L}$, an elevated serum betahydroxylbuterate/acetoacetate level greater than $5 \mathrm{mmol} / \mathrm{L}$ and dehydration. Insulin deficiency is the main precipitating factor (1). It is a medical emergency and needs immediate and prompt diagnosis and early management $(1,2)$. Euglycemic DKA is defined as ketoacidosis $(\mathrm{pH}<7.3$ or serum bicarbonate $<18$ $\mathrm{mmol} / \mathrm{L}$ ), an elevated serum betahydroxylbuterate/ acetoacetate level greater than the upper limit of reference range of $0.02-0.27 \mathrm{mmol} / \mathrm{L}$ with normal plasma glucose or a milder degree of hyperglycemia of between 200 to $250 \mathrm{mg} / \mathrm{dl}$ (3). Normoglycemia in euglycemic DKA can cause diagnostic difficulty especially in resource constrained countries where laboratory support is not readily available (4). Moreover, the clinical features of DKA can be concealed by co-existing infections such as malaria and septicemia (5-7) which are more common in Africa and can result in delayed diagnosis or misdiagnosis with lethal consequences.

Euglycemic DKA has not been previously reported in Ghana in the literature. We present 2 cases of euglycemic DKA both of which were precipitated 
by infections and diagnosis of euglycemic DKA was delayed in both of them.

\section{Case Report}

\section{Case 1}

A 13-year-old girl who had been diagnosed with type 1 diabetes mellitus (T1DM) for the past one year was sent to a hospital with fever, bodily weakness, nausea, vomiting and poor feeding for 6 days. She was treated at home for malaria with artemeter/lumefantrene but she vomited all medication and did not improve. She did not have history of previous admissions and surgery and no family history of diabetes mellitus.

On examination, she was wasted (weight 30 kilograms: below the 3rd percentile, height 145 centimeters, on the 10th percentile), febrile (temperature - $38.7{ }^{\circ} \mathrm{C}$ ), lethargic, drowsy but conscious. She was also breathless with respiratory rate of 56 cycles per minute, dehydrated with loss of skin turgor (level of dehydration was estimated to be $10 \%$ ). Her blood pressure (BP) was $80 / 50 \mathrm{mmHg}$, and her pulse was 110 beats per minute. Tanner staging was 2 for both pubic hair and breast development.

\section{Investigations were as follows:}

Rapid diagnostic test (RDT) for malaria was positive, blood smear for malaria parasites was positive with $3+$. Other investigations revealed; normal complete blood count (hemoglobin $13.9 \mathrm{~g} / \mathrm{dl}$ ), Potassium - $3.7 \mathrm{mmol} / \mathrm{L}$, Sodium - $140.0 \mathrm{mmol} / \mathrm{L}$, blood glucose - $97.5 \mathrm{mg} / \mathrm{dl}$ and HbA1c- 10.3\%. Urinalysis was negative for both nitrite and leucocyte esterase activity, blood and urine cultures yielded no bacteria growth. She was diagnosed as severe malaria with shock.

She was put on intranasal oxygen, $6 \mathrm{ml} / \mathrm{L}$, intravenous (IV) artesunate and was resuscitated with $600 \mathrm{ml}$ of normal saline over 30 minutes which improved the peripheral circulation. She was then put on $5 \%$ dextrose saline at maintenance rate. The following day on review, she was in coma, afebrile (temperature was $36.9^{\circ} \mathrm{C}$ ), respiratory rate 38 cycles per minute, deep sighing, air entry was good, chest was clear bilaterally with no crackles. Brachial pulse was 90 beat/minute, capillary refill time was less than 2 seconds, heart sounds normal, BP 106/58 $\mathrm{mmHg}$.

\section{Investigations}

Her blood glucose was $185.4 \mathrm{~g} / \mathrm{dl}$, urine ketone 3+, urinalysis was negative of nitrite and leucocyte esterase activity.

Diagnosis was changed to malaria induced euglycemic DKA.

Intravenous (IV) artesunate and IV 5\% dextrose saline at maintenance were continued.

Euglycemic DKA was managed using ISPAD protocol (8). She was given IV short-acting human insulin at a dose of 0.1 units per kilogram (3 units per hour). Potassium chloride was also started at the same time as the short-acting human insulin at a dose of $40 \mathrm{mmol} / \mathrm{L}$, administered via the IV fluids. She was monitored and reviewed every hour for blood glucose, every two hours for urine ketones using urine dipstick test, and every 4 hours for electrolytes and vital signs. Twelve hours after continuous IV insulin administration, coma score had improved and she could open her eyes and responded weakly to calls.

All ketones cleared from urine after 36 hours of continuous IV insulin administration. Blood glucose level remained between $93.6 \mathrm{mg} / \mathrm{dl}$ to 190.8 $\mathrm{mg} / \mathrm{dl}$.

All signs and symptoms resolved by 5 days after admission and she was well and self-caring. Intravenous insulin was changed to subcutaneous injection using multiple dose regimen consisting of short-acting and intermediate-acting human insulin.

\section{Case 2}

A 12-year-old girl with T1DM diagnosed 2 years ago was brought to a district hospital with fever, decreased level of consciousness and difficulty breathing. She developed fever, nausea, vomiting and poor feeding four days prior to presentation. She had been treated with over the counter med- 
ication at home but she worsened and so she was brought to hospital. She was attendant at a diabetes clinic and was on multiple dose insulin injection regimen using short-acting human insulin and intermediate- acting human insulin.

On examination, she weighed $38 \mathrm{~kg}$ (above 10th percentile), height was $145 \mathrm{~cm}$ (below 25th percentile), she was febrile (temperature - 39.2oC), unconscious, had deep labored respiration, but was not dehydrated. Her blood pressure was 100/60 mmHg, and her pulse was 110 beats per minute. Respiration was 24 cycles per minute, air entry was good, breath sounds were clear with no crackles bilaterally. Her Tanner staging was 2 for breast development and 3 for pubic hair.

\section{Investigations were as follows:}

Blood glucose was $147.6 \mathrm{mg} / \mathrm{dl}$, blood smear for malaria parasites was positive with $2+$, complete blood count was otherwise normal except hemoglobin of $10.7 \mathrm{~g} / \mathrm{dl}$. Her Potassium was $4.1 \mathrm{mmol} / \mathrm{L}$ and Sodium was $138.0 \mathrm{mmol} / \mathrm{L}$. Blood gases and serum ketones could not be done, urinalysis revealed positive nitrite and leucocyte esterase activity but urine and blood cultures yielded no bacteria growth. She was diagnosed as cerebral malaria and urinary tract infection.

She was put on intranasal oxygen, $4 \mathrm{ml} / \mathrm{L}$, IV gentamycin and cefuroxime $100 \mathrm{mg}$ tds, IV artesunate and was put on $70 \%$ of maintenance of $5 \%$ dextrose saline. After two days she was comatose. Blood smear for malaria was negative but rapid diagnostic test for malaria was positive. Urinalysis was still positive for nitrite and leucocyte esterase. Urine ketone revealed 4+ of ketones, blood glucose was $196.2 \mathrm{mg} /$ dl. The diagnosis was changed to euglycemic DKA precipitated by infection.

Nasogastric tube was put in and oral artemeter/ lumefantrene was given for three days, antibiotics were changed to IV ceftriaxone $3 \mathrm{~g}$ daily for 7 days and she was also managed for diabetic ketoacidosis according to ISPAD protocol (8). She opened her eyes 18 hours after starting DKA management and she had significantly improved by the 6th day after admission and all ketones had cleared from the urine. She was completely well and self-caring by the 7 th day of admission.

\section{Discussion}

Euglycemic DKA is a clinical triad comprising of increased anion gap metabolic acidosis, ketonemia or ketonuria and normal blood glucose level less than $250 \mathrm{mg} / \mathrm{dl}$ (3). Euglycemic DKA is known to be caused by gestational diabetes, reduced calorie intake, excessive alcohol consumption, use of cocaine, pancreatitis, and chronic hepatitis (9). Although euglycemic DKA has been reported in SARs-CoV-2 infection in adult patients with type 2 diabetes mellitus on sodium-glucose cotransporter 2 inhibitors (SGLT-2 inhibitors) (10,11), it has not been described in children with diabetes in Ghana. Nathan Morrison et al (10) reported on a man in his 40 s with type 2 diabetes who developed severe COVID-19 and subsequently was diagnosed with euglycemic DKA. Vitale et al (11) also described 5 patients with type 2 diabetes patients who developed euglycemic DKA following SARs-CoV-2 infection. All the patients were being managed with SGLT2 inhibitors.

Infection induced metabolic acidosis has similar clinical features as metabolic acidosis in DKA $(1,2,6,12)$ and this can mask diagnosis in euglycemic DKA leading to delayed diagnosis or misdiagnosis. Patient 1 had severe malaria with euglycemic DKA whereas patient 2 had severe malaria and UTI with euglycemic DKA. The diagnosis of euglycemic DKA was missed in both cases at presentation and were only considered later on review because patients did not improve on antimalarial and antibiotics only. What makes diagnosis more difficult in Ghana and other resource limited countries is lack of laboratory support (4). Blood ketone and blood gases levels are not done in almost all hospitals in Ghana, therefore, they were not done for our patients. However, history of type 1 diabetes coupled with clinical features of metabolic acidosis such as weakness, bodily pains and Kussmaul breathing as well as ketonuria in presence of normoglycemia raised suspicion of euglycemic DKA in our patients.

In patients with type 1 diabetes who are not able to maintain sufficient carbohydrate intake such as 
in state of fasting (7) or in nausea, vomiting and poor feeding caused by infections, severe ketoacidosis with normal blood glucose level can occur as a consequence of continuing insulin administration. This in turn leads to decreased gluconeogenesis and improved glucose utilization at the cellular level $(3,7)$. In both our patients, poor feeding from nausea and vomiting more likely precipitated euglycemia with ketonemia which was detected as significant ketonuria. The difficulty was the fact that they had infections which are more common in medical practice in Ghana and therefore caused delay in diagnosis. Euglycemic DKA are undiagnosed, especially in the resource poor setting, because blood gas analysis and ketonemia tests are not freely available in Ghana and other resource constrained countries. Therefore, training in the form of workshops and continuous professional developments should be organized on regular basis for doctors and other health workers to improve on health workers' awareness of euglycemic DKA. Protocols and guidelines should be provided to ensure that all critically ill children especially those with type 1 diabetes on admission at emergency units and wards in resource limited countries should be assessed for euglycemic DKA by checking blood glucose and urine ketones levels.

\section{Conclusions}

Although it may be challenging to diagnose because of normal or only slightly elevated blood glucose levels, it is important to consider euglycemic DKA in the appropriate clinical setting as a differential diagnosis when evaluating a patient with clinical features of metabolic acidosis. In resource limited countries, high degree of suspicion should be elicited in diagnosis of euglycemic DKA for those who have a history of diabetes or previous history of clinical features suggestive of diabetes and raised urine ketones despite normal blood glucose values.

\section{Declarations}

\section{Ethics approval and consent to participate}

The patients' legal guardians gave consent for publication of clinical data. Need for ethics committee approval was non-applicable as study consists of only two patients. Patients and parents gave written informed consent prior to manuscript writing.

\section{Consent for publication}

Written consent for publication was obtained from patients and their legal guardians. Copies of written informed consent have been included in manuscript submission.

\section{Conflict of interest}

The authors declare that they have no competing interests.

\section{References}

1. Westerberg DP. Diabetic Ketoacidosis: Evaluation and Treatment. Am Fam Physician. 2013;87(5):33746.

2. Perilli G, Saraceni C, Daniels MN, Ahmad A. Diabetic Ketoacidosis: A Review and Update. Curr Emerg Hosp Med Rep. 2013;1:10-17.

3. Modi A, Agrawal A, Morgan F. Euglycemic Diabetic Ketoacidosis: A Review. Curr Diabetes Rev. 2017;13(3):315-321.

4. Petti CA, Polage CR, Quinn TC, Ronald AR, Sande MA. Laboratory Medicine in Africa: A Barrier to Effective Health Care. Clinical Infectious Diseases. 2006;42(3):377-82.

5. Misra UK, Kalita J, Prabhakar S, Chakravarty A, Kochar D, Nair PP. Cerebral malaria and bacterial meningitis. Ann Indian Acad Neurol. 2011;14(suppl 1):S35-S39.

6. Cruz AT, Lane RD, Balamuth F, Aronson PL, Ashby DW, Neuman MI et al. Updates on pediatric sepsis. JACEP Open. 2020;1(5):981-93.

7. Thawabi M, Studyvin S. Euglycemic Diabetic Ketoacidosis, a Misleading Presentation of Diabetic Ketoacidosis. North American Journal of Medical Sciences. 2015;7(6):291-94.

8. Wolfsdorf JI, Glase N, Agus M, Fritsch M, Hanas $\mathrm{R}$, Rewers A, Sperling MA, Codner E. ISPAD Clinical Practice Consensus Guidelines 2018: Diabetic ketoacidosis and the hyperglycemic hyperosmolar state. Pediatric Diabetes. 2018;19(Suppl. 27):155-177. 
9. Barski L, Eshkoli T, Brandstaetter E, Jotkowitz A. Euglycemic diabetic ketoacidosis. Eur J Intern Med. 2019;63:9-14.

10. Morrison N, Barnett K, Tantum J, Morrison HK, Whalen M. A Case of Euglycemic Diabetic Ketoacidosis in a Patient With Type 2 Diabetes Mellitus and COVID-19. Cureus. 2020;12(12): e12029. doi:10.7759/cureus.12029
11. Vitale RJ, McDonnell ME, Palermo NE, Fisher NDL. Euglycemic Diabetic Ketoacidosis With COVID-19 Infection in Patients With Type 2 Diabetes Taking SGLT2 Inhibitors. AACE Clin Case Rep. 2021; 7(1): 10-13.

12. Possemiers H, Vandermosten L, Van den, Steen PE. Etiology of lactic acidosis in malaria. Plos Pathog. 2021 Jan;17(1):1-17. 